\title{
The effect of extended acquisition upon the replacement capacity of intertrial reinforcement'
}

PETER A. PRATT and JOSEPH HALPERN, University of Denver, Denver, Colo. 80210

A modified extinction procedure was used to determine whether simple repeated exposure to intertrial reinforcement (ITR) was sufficient for loss of ITR replacement capacity. This modified extinction consisted of interpolating ITRs between extinction trials. Extinction performance suggested that ITR remained effective despite extended exposure during acquisition.

Recent experimental evidence suggests that characteristic stimuli are produced by reward (R) and nonreward (N) (Capaldi, 1966). According to Capaldi, $\mathrm{R}$ and $\mathrm{N}$ trials in an instrumental learning task occasion internal proprioceptive stimulus aftereffects, SR and SN, respectively. These hypotheses concerning differential stimulus aftereffects form the basis of an explanation for the partial reinforcement effect (PRE). Specifically, for any partial reinforcement schedule, a construct, N-length, is defined as the number of consecutive $\mathrm{N}$-trials prior to an R-trial. It is assumed that an R-trial following any series of $\mathrm{N}$ trials serves to condition the aftereffect of nonreward to the instrumental response, RI. Habit strength which has accrued over several NR transitions for any particular N-length, e.g., SN-RI, is assumed to generalize over $\mathrm{N}$-lengths longer than those involved in the original conditioning. Further, SN connections are assumed to generalize over a larger span of trials than SR connections. The result is that continuous reinforcement results in only SR connections which generalize over shorter SNs in extinction than partial reinforcement which results in both SR and SN connections.

It follows from the theory that anything interfering with the establishment of SN-RI would result in the elimination of the PRE. Capaldi, Hart, \& Stanley (1963) employed an intertrial reinforcement (ITR) procedure in a straight alley learning situation to interfere with the establishment of the SN-RI. This ITR procedure consisted of placing the rat in a baited goal box during the intertrial interval (ITI). It was assumed that an ITR following an $\mathrm{N}$-trial would replace $\mathrm{SN}$ with SR. This procedure could not result in SN-RI since RI does not occur. Capaldi et al employed three groups of rats; a single continuously reinforced group (Group C) and two groups receiving a 50\% reinforcement schedule. The 50\% groups differed in that ITRs were administered between NR transitions (N-ITR-R), Group PN, and between RN transitions, Group PR. All groups received 30 acquisition trials followed by 20 extinction trials. The results showed that Group PN was no more resistant to extinction than Group $C$ while Group PR was significantly more resistant to extinction than the other groups. The relatively rapid extinction of Group PN was ascribed to a failure of $\mathrm{SN}$ to become conditioned to the locomotor response because of the replacement caused by ITR.

Subsequent investigations (Black \& Spence, 1965; Spence, Platt, \& Matsumoto, 1965) established that the PRE could be eliminated only with approximately 30 or fewer acquisition trials. Extended acquisition (70 or more trials) resulted in reinstatement of the PRE. Two possible explanations for the above have been proposed. The first involves some statement to the effect that the PRE comes to be regulated increasingly by frustration with extended training. The second involves an assumption that Ss learn to discriminate between $R$ and ITR trials. Capaldi \& Olivier (1967) attempted to determine which of these two interpretations of the failure of ITR to eliminate the PRE after extended training was more appropriate. Although Capaldi and Olivier's (1967) study supported the inference that the failure of ITR to eliminate the PRE following extended training was related to the repeated use of ITR and not to the number of rewarded trials as such, it provided no direct evidence to that effect.

The purpose of the present experiment was to test whether repeated exposure to ITR was a sufficient condition for loss of its replacement capacity. For this purpose, a modified extinction procedure was developed. This modified extinction consisted of interpolating ITRs between extinction trials. The rationale was that if ITR were replacing $S N$ with $S R$, the successive increase in SN magnitude occasioned by each extinction trial could be interrupted. Since Capaldi (1966) assumed that extinction was a function of the generalization of habit strength for S-RI connections over SNs of greater magnitude than those encountered during acquisition, the interruption of the successive increase in SN magnitude should also interrupt the generalization of habit strength for any S-RI connection. Therefore, by administering ITRs between appropriate extinction trials, the generalization of habit strength for a particular SN-RI connection could be retarded. Thus, whether or not ITR was replacing SN with SR could be determined from a comparison of regular with modified extinction. If modified extinction Ss were significantly more resistant to extinction than regular extinction Ss, ITR was performing its replacement function. If modified extinction Ss were no more resistant to extinction than regular extinction Ss, ITR had lost its replacement capacity prior to the onset of modified extinction trials.

\section{SUBJECTS}

The Ss were 48 experimentally naive, male albino rats of the SpragueDawley strain. They ranged in age from 100 to 130 days at the beginning of the experiment.

\section{APPARATUS}

The apparatus, a straight alley runway, has been previously described by Capaldi et al (1963). It differed only in the following respects. Running times were measured with a Hunter Digital Display Timer. After raising the door of the start box, the timer was started by $S$ interrupting a photobeam located $1 \mathrm{in}$. beyond the door of the start box, and the timer was stopped when $S$ interrupted a photobeam located 1 in. in front of the goal area. The distance be tween the two photobeams was 82 in. A wire mesh cage served to contain Ss between trials.

\section{PROCEDURE}

Ad lib feeding was discontinued three days prior to the beginning of pretraining. Thereafter, all $S s$ were maintained on a daily ration of approximately $12 \mathrm{~g}$ of Purina Lab Chow.

Five days of pretraining were administered. The rats were handled in groups of five for $15 \mathrm{~min}$ on the first day. On Day 2, the rats were given additional handling plus $5 \mathrm{~min}$ of free exploration in the alley. On Day 3, each rat was placed in the baited goal box until observed to eat from the food cup filled with $.045 \cdot \mathrm{g}$ Noyes pellets. Day 4 completed goal box training as each $S$ was given four placements in the goal box baited with six Noyes pellets. Each rat was given one $R$ trial on the fifth day.

Prior to acquisition training, the Ss were randomly assigned to four groups of 12 rats each. The groups were distinguished as a function of whe ther or not ITRs were administered during acquisition and extinction, with $\mathrm{A}=$ acquisition, $\mathrm{E}=$ extinction, $1=\mathrm{ITR}$ absent, $2=\mathrm{ITR}$ present. The four groups, then were A1E1, A1E2, A2E1, and A2E2.

On the sixth day, the groups began 72 trials of extended acquisition training. Nine trials per $S$ per day were run according to the schedule RRNNRNNRR on Days 6, 8, 10, and 12, and RNNRRNNRR on Days 7,9, 11, and 13. Groups A2E 1 and A2E2 received ITRs after Trials 2 and 5 in the first schedule, and after Trials 1 and 5 in the second schedule. Thus, the 
A2 groups received ITRs only between $R$ and $N$ trials. On $R$ trials, $S$ received six $.045-\mathrm{g}$ Noyes pellets and was removed from the goal box after the pellets were consumed. On nonreinforced trials, $S$ was removed $10 \mathrm{sec}$ after goal box entry. The intertrial interval was $30 \mathrm{sec}$. When an ITR was administered, the first $15 \mathrm{sec}$ of the ITI was spent in the retaining cage, whereupon $S$ was placed in the baited goal box for the remainder of the ITI. $S$ was then placed in the start box for the next trial.

Upon completion of acquisition training, Groups A1E1 and A2E1 were given 30 regular extinction trials ( 10 per day), while Groups A1E2 and A2E2 were given 30 modified extinction trials ( 10 per day). Modified extinction involved giving an ITR after the second, fourth, six th, eighth, and tenth extinction triałs.

\section{RESULTS AND DISCUSSION}

The mean running time on the last day of acquisition was converted to speed, and a single factor analysis of variance with each of the four groups representing a treatment level showed no significant between-group difference. Thus, all groups attained comparable levels of acquisition despite the fact that A2 Ss received repeated exposure to ITR.

The mean running time per block of 10 extinction trials was converted to speed for each $S$ and Fig. 1 provides the average speed per group in three blocks of 10 trials each. Prior to any direct reference to the figure, it is helpful to stress that those Ss receiving acquisition ITRs received them between $R$ and $N$ trials (similar to the PR group of Capaldi et al, 1963). A PR rather than a PN procedure was used in order that Ss would receive repeated exposure to ITR without it performing its replacement function. By using identical reinforcement schedules for all groups with half of the groups receiving ITRs between $R$ and $N$ trials and half of the groups receiving no ITRs, groups differed only with respect to exposure vs no exposure to ITR at the end of acquisition. Consequently, if mere repeated exposure were a sufficient condition for loss of replacement capacity, then Group A IE2 should have been more resistant to extinction than Group A2E2. Inspection of Fig. 1 shows that this was not the case. Ordinarily, this finding alone would provide support for the contention that mere exposure alone was not sufficient to result in a loss of replacement capacity. However, the data for Group A1E2 are somewhat confounded in that these Ss were observed to engage in competing responses in the vicinity of the start box (appearing to seek additional ITRs) on extinction trials following ITRs. The improvement in performance across blocks for Group A IE2 relative to the other groups, can be attributed to the gradual elimination of this behavior. In fact, a comparison of running speed for A IE2 with that of AIEI and A2E 1 showed that $\mathrm{A} 1 \mathrm{E} 2$ was significantly more resistant to extinction in the third trial block, $\mathrm{t}(33)=2.21, \mathrm{p}<.05$. This competing response tendency in Group A2E2 had long since been eliminated during the course of acquisition.

Clearly then, the above cursory treatment of the data was not sufficient to determine whether mere repeated exposure was adequate for loss of replacement capacity. However, an analysis of variance with $A, E$, and trials (T) serving as the independent variables and speed as the dependent variable resulted in significant $T$ and $E$ main effects, $F(2,88)=154.26, p<.01$ and $F(1,44)=5.09, p<.05$. The significant $T$ main effect demonstrates the extinction that is obvious from the figure. Of more immediate relevance was the significant $E$ main effect due primarily to Group A2E2. Specifically, there was an effect due to ITR during modified extinction in this group. Therefore, repeated exposure to ITR during acquisition was not a sufficient condition for loss of replacement capacity.

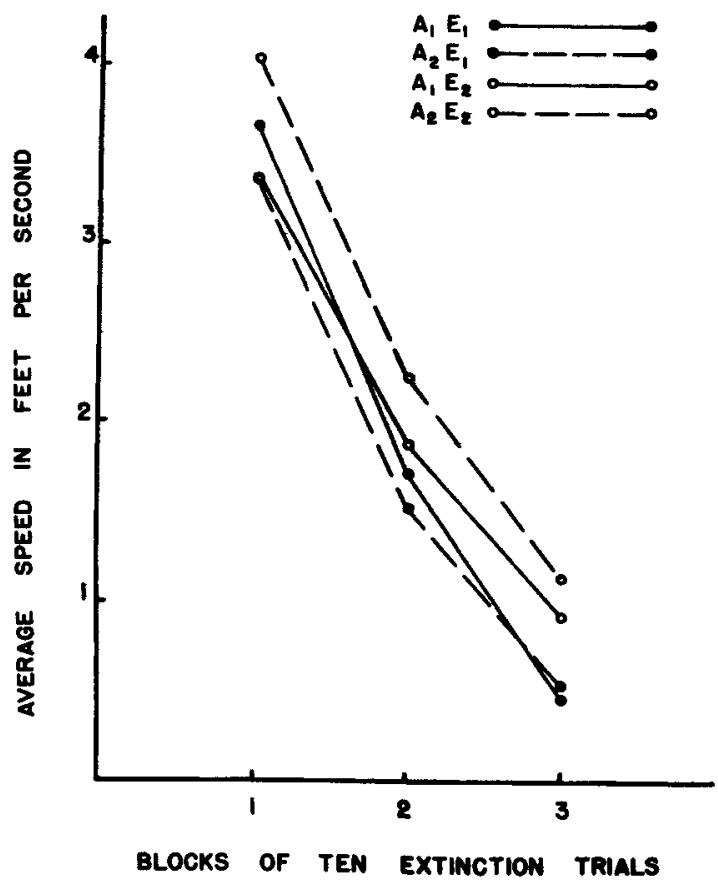

Fig. 1. Average speed per groups as a function of blocks of 10 extinction trials.

With the respect to the discrimination hypothesis (ITR loses its replacement capacity as a function of extended experience because a discrimination between $R$ and ITR trials is learned), the results imply one of two things. If simple repeated exposure to ITR in conjunction with $R$ and $\mathbf{N}$ trials provided a sufficient condition for the development of a discrimination, then Group A2E2 would have been no more resistant to extinction than the $E 1$ groups, i.e., ITR would have lost its effectiveness. Thus, the results did not support a discrimination hypothesis. However, it is plausible that the ITR following an $\mathrm{R}$ trial was largely ignored, i.e., operated as a continuation of the $R$ trial reinforcement. If the latter is true, then the results are irrelevant with respect to the discrimination hypothesis.

\section{REFERENCES}

BLACK, R. W., \& SPENCE, K. W. Effects of intertrial reinforcement on resistance to extinction following extended training. Journal of Experimental Psychology, 1965, 70, 559-563.

CAPALDI, E. J. Partial reinforcement: An hypothesis of sequential effects. Psychological Review, 1966, 73, 459-477.

CAPALDI, E. J., HART, D., \& STANLEY, L. R. Effect of intertrial reinforcement on the aftereffect of nonreinforcement and resistance to extinction. Journal of Experimental Psychology 1963, 65, 70-74.

CAPALDI, E. J., \& OLIVIER, W. P. Effect of intertrial reinforcement following a substantial number of consistently rewarded trials. Journal of Experimental Psychology, 1967, 75, 135-138.

SPENCE, K. W., PLATT, J. R., \& MATSUMOTO, R. Intertrial reinforcement and the partial reinforcement effect as a function of number of training trials. Psychonomic Science, 1965, 3, 205-206. NOTE

1. This research was supported in part by National Institute of Mental Health Grant MH 14102-01. 